\title{
KYMLICKA EN PRO DE UNA CIUDADANÍA DIFERENCIADA
}

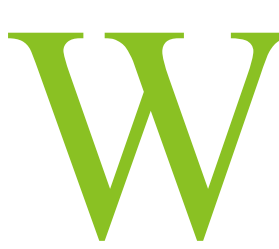

ill Kymlicka, director de investigaciones del Centro Canadiense de Filosofía y Política Pública del Departamento de Filosofía de la Universidad de Ottawa, es autor de otros conocidos libros como Liberalism, Community and Culture (1989) y Contemporary Political Philosophy (1991), traducido en editorial Ariel con el título Filosofía política contemporánea (1995).

En Ciudadanía multicultural ${ }^{1}$, Kymlicka realiza un análisis riguroso y equilibrado de la integración de las minorías culturales en sociedades con una cultura mayoritaria dominante. Asombra que un investigador medianamente joven se enfrente a un tema tan cargado de apasionamientos con una serenidad intelectualmente envidiable. Su exposición es pedagógica y clara. Deslinda y define bien los conceptos, argumenta siguiendo un hilo conductor y concluye con coherencia.

Con independencia de que se compartan o no algunas de sus opiniones, buena parte del libro se nutre de datos históricos documentados que contribuyen a aumentar los conocimientos de cualquier persona. De modo que es una buena terapia para dos tipos de enfermedades: la de aquellos que exageran el derecho a su cultura y no respetan los derechos de los demás, y la de quienes tienen fobia a las diferencias.

Kymlicka parte de una teoría liberal del Estado, pero a la vez acepta la importancia que tiene la cultura en el desarrollo de la personalidad de cada individuo. Aboga en consecuencia por la existencia y la defensa de la identidad cultural de los grupos sociales o de los pueblos.

Conviene aclarar que el liberalismo que Kymlicka defiende no es el que hoy se identifica con el neoliberalismo económico. Cuando a lo largo del

${ }^{1}$ KYMLICKA, WILL. Ciudadanía Multicultural. Ed. Paidós, 303 pp. Barcelona. 1996. Original Multicultural Citizenship. Oxford University Press. Oxford. 1995. 280 pp. 
texto se habla de «principios liberales», en muchas ocasiones ese término equivale a defender los derechos civiles individuales, que fueron reclamados por el liberalismo, y que hoy están recogidos en la mayoría de las constituciones occidentales. Este liberalismo insiste en que la base de las sociedades democráticas modernas es el respeto a la libertad del individuo.

La novedad de esta obra radica en que Kymlicka intenta demostrar que la teoría política liberal no debe defender sólo los derechos de los individuos, sino también los derechos de los diferentes grupos culturales. Hasta ahora muchos liberales han visto en esto una oposición o una incompatibilidad. En cambio, Kymlicka sostiene que dentro de los derechos del individuo se encuentra el derecho a mantener o elegir la cultura en la que quiere desarrollar su vida; un elemento esencial es el lenguaje que cada grupo humano utiliza: «Una teoría liberal de los derechos de las minorías debe explicar cómo los derechos de las minorías coexisten con los derechos humanos, y también cómo los derechos de las minorías están limitados por los principios de la libertad individual, democracia y justicia social. Tal explicación constituye justamente el objetivo de este libro» (p. 19).

Para deslindar campos, Kymlicka distingue entre Estados «multinacionales» (donde la diversidad cultural surge de la incorporación de culturas que anteriormente poseían autogobierno y estaban concentradas territorialmente a un Estado mayor) y Estados «poliétnicos» (donde la diversidad cultural surge de la inmigración individual y familiar). Y a partir de ahí explica la distinta situación de «minorías nacionales» (en Estados multinacionales) y «grupos étnicos» (en Estados poliétnicos) (pp. 19-20).

Las primeras se caracterizan por ser grupos culturales preexistentes en un territorio concreto, y que son invadidos contra su voluntad, o pasan a formar parte de otra nación con otra cultura mayoritaria, como consecuencia también de un proceso de federalismo o por distintos acuerdos.

En la inmigración, en cambio, un individuo o un grupo familiar deciden cambiar de nación y se trasladan a un país de cultura diferente, pero esto lo hacen voluntariamente, eligen ese país, nadie les obliga a ello. Luego la reclamación de sus derechos culturales es diversa a la del primer caso. Los inmigrantes no tienen derecho a exigir el autogobierno, pero sí que tienen derecho a exigir un respeto institucional y legal a la expresión de su propia identidad.

\section{En pro de una ciudadanía diferenciada}

Algunos liberales han mantenido que así como el Estado liberal mantiene la separación entre Estado y religión, del mismo modo el Estado liberal debe construirse de espaldas a las diversas culturas a las que sus ciudadanos pertenecen, es decir sin distinguir entre sus ciudadanos por razón de su pertenencia 
a un determinado grupo cultural. El ciudadano liberal sería en la vida pública un ciudadano neutral, y sólo reflejaría su pertenencia cultural en su vida privada. Utilizando el lenguaje del autor, es lo que se ha denominado «una omisión bienintencionada».

Esto, dirá Kymlicka, es una utopía que no responde a la realidad. Además de los derechos comunes de todos los ciudadanos es posible defender la necesidad de una ciudadanía diferenciada, según la cual el Estado tiene obligación de adoptar «medidas específicas» en función de la pertenencia grupal orientadas a acomodar las diferencias nacionales y étnicas.

Existen, al menos, tres formas de derechos diferenciados en función de la pertenencia a un grupo:

(1) derechos de autogobierno (la delegación de poderes a las minorías nacionales, a menudo a través de algún tipo de federalismo);

(2) derechos poliétnicos (apoyo financiero y protección legal para determinadas prácticas asociadas con determinados grupos étnicos o religiosos);

(3) derechos especiales de representación (escaños garantizados para grupos étnicos o nacionales en el seno de instituciones centrales del Estado que los engloba) (p. 20).

La reclamación del pensador canadiense es muy clara; a los grupos nacionales o grupos con una etnicidad específica se les debe reconocer una identidad política permanente con un estatus constitucional.

Existe una diferenciación permanente en los derechos o estatus de los miembros de ciertos grupos. Es necesario su reconocimiento para evitar que esas diferencias culturales no se pierdan o desaparezcan.

Las teorías tradicionales de los derechos humanos no han dado una solución a esta cuestión. La propia Declaración Universal de los Derechos Humanos de la ONU no reconoció ningún derecho relacionado con los derechos étnicos o de minorías nacionales.

\section{El valor de la pertenencia grupal}

Kymlicka intenta hacer ver que la cultura no es un sobreañadido a los derechos individuales de la persona, sino que está intrínsecamente unida a la libertad del individuo, es decir también sería antiliberal imponer una cultura a un individuo o a un grupo, usando la fuerza y contra la voluntad de los mismos. Si la cultura no fuera un valor, sería más cómodo y muchas veces más barato homogeneizar. Por lo tanto, hay que garantizar la identidad cultural dentro del marco del liberalismo. «Los principios básicos del liberalismo son principios de libertad individual. Los liberales únicamente pueden aprobar los derechos de las minorías en la medida en que éstos sean consistentes con el respeto a la libertad o autonomía de los individuos» (p. 111). Kymlicka demuestra que los derechos de las minorías no sólo son 
compatibles con la libertad individual, sino que pueden de hecho promoverla porque la causa de la libertad muchas veces encuentra sus bases en la autonomía de un grupo nacional. (Capítulo V, Libertad y cultura).

A lo largo de la historia las minorías han sido maltratadas en distintos grados, que van desde la eliminación física, la expulsión, la limpieza étnica, el genocidio, o fueron asimiladas por la fuerza, o segregadas, sin concederles los derechos del resto de la población. Al negar estos derechos además de cometer una injusticia lo único que se ha logrado es exacerbar los conflictos etnoculturales: «Así mayorías y minorías se enfrentan cada vez más respecto de temas como los derechos lingüísticos, la autonomía regional, la representación política, el currículum educativo, las reivindicaciones territoriales, la política de inmigración y naturalización, e incluso acerca de símbolos nacionales, como la elección del himno nacional y de las festividades oficiales» (p 13).

Pretender la separación entre Estado y cultura es absurdo. Hoy más que nunca las sociedades liberales deben responder a cuestiones relacionadas con las minorías culturales, entre las que Kymlicka señala:

«¿Qué lenguas deberían aceptarse en los Parlamentos, burocracias y tribunales?, ¿se deberían dedicar fondos públicos para escolarizar en su lengua materna a todos los grupos étnicos o nacionales?, ¿se deberían trazar fronteras internas (distritos legislativos, provincias, Estados) tendentes a lograr que las minorías culturales formen una mayoría dentro de una región local?, ¿debería devolver poderes gubernamentales el nivel central a niveles locales o regionales controlados por minorías concretas, especialmente en temas culturalmente delicados como la inmigración, las comunicaciones y la educación?, ¿deberían distribuirse los organismos políticos de acuerdo con un principio de proporcionalidad nacional o étnica?, ¿se deberían conservar y proteger las zonas y lugares de origen tradicionales de los pueblos indígenas para su exclusivo beneficio, protegiéndoles de la usurpación de los colonos o de los explotadores de recursos?, ¿qué grado de integración cultural puede exigirse de los inmigrantes y los refugiados antes de que adquieran la ciudadanía?» (pp. 17-18).

\section{Los derechos diferenciados en función del grupo. Restricciones internas y protecciones externas}

Según el profesor canadiense, los procedimientos vinculados a los derechos humanos no son capaces de resolver estas controvertidas cuestiones. Por eso él defiende la necesidad de formas de ciudadanía en función del grupo.

Especialmente esclarecedor resulta el análisis que Kymlicka presenta sobre la interminable discusión entre defensores y detractores de los derechos 
colectivos. Esta noción resulta para el pensador canadiense confusa e innecesaria. A su juicio es más apropiado hablar de derechos especiales o derechos diferenciados en función del grupo. Este concepto rompe con la supuesta incompatibilidad o conflicto entre derechos individuales y derechos de determinados grupos culturales o colectivos, y en otras ocasiones con la supuesta necesidad de su jerarquización.

Muchos liberales temen que los derechos colectivos reivindicados por los grupos étnicos y nacionales vayan en contra de los derechos individuales. Para aclarar esta cuestión, Kymlicka distingue entre dos tipos de reivindicaciones que un grupo podría hacer.

Por una parte, para preservar su identidad algunos grupos étnicos o nacionales pretenden imponer algunas restricciones internas a los componentes de ese grupo (relaciones intragrupales). Pretenden usar el poder del Estado para restringir la libertad de sus propios miembros en nombre de la solidaridad del grupo o la pureza cultural («restricciones internas»). «Esto plantea el peligro de la opresión individual» (p. 59). Aquí las libertades civiles y políticas básicas de los miembros del grupo se ven restringidas.

Otros, sin embargo, exigen el derecho de un grupo a limitar el poder político y económico ejercido sobre dicho grupo por la sociedad de la que forma parte, con el objeto de asegurar que los recursos y las instituciones de las que depende la minoría no sean vulnerables a las decisiones de la mayoría («protecciones externas» que implican relaciones intergrupales). Esto plantea el problema de la justicia o injusticia entre grupos. «El primer tipo de restricciones implica la reivindicación de un grupo contra sus propios miembros; el segundo implica la reivindicación de un grupo contra la sociedad en la que está englobado... El primer tipo de restricciones tiene el objetivo de proteger al grupo del impacto desestabilizador del disenso interno (por ejemplo, la decisión de sus miembros de no seguir las prácticas o las costumbres tradicionales)... mientras que el objetivo del segundo es proteger al individuo del impacto de las decisiones externas (por ejemplo, las decisiones políticas y económicas de la sociedad mayor)» (p. 58).

Kymlicka piensa que el segundo tipo, «las protecciones externas», no entran en conflicto con los principios liberales que protegen la libertad individual. Estas protecciones «únicamente son legítimas en la medida en que fomentan la igualdad entre los grupos, rectificando las situaciones perjudiciales o de vulnerabilidad sufridas por los miembros de un grupo determinado» (p. 212).

Las protecciones externas, es decir, «la concesión de derechos especiales de representación, de reivindicaciones territoriales o de derechos lingüísticos a una minoría no necesita, y muchas veces no implica, una posición 
de dominio sobre otros grupos» (p. 60).Esto último no va contra la justicia, sino que puede ser un derecho compatible con el liberalismo y con los derechos individuales.

Estos derechos diferenciados en función del grupo, que ya han sido citados, son los derechos de autogobierno, derechos poliétnicos y derechos especiales de representación (cf. p. 61). Protegen a un grupo cultural frente a otro grupo, en general mayoritario, pero a su vez respetan los derechos individuales de los componentes del grupo.

Sin embargo no ocurre lo mismo con las restricciones internas. Las políticas públicas de países liberales no han aprobado nunca legalmente imposiciones de costumbres que vayan contra las Cartas de Derechos Humanos, tales como matrimonios forzados $\mathrm{u}$ acordados, la clitoridectomía, la supresión del derecho a la propiedad por conversión religiosa, el reconocimiento de los divorcios mediante el talaq, costumbre islámica de repudio de la mujer, el sometimiento forzado a ceremonias de iniciación en tribus indias, la discriminación de las niñas en materia educativa, la prohibición a las mujeres del derecho al voto o al ejercicio del poder, etc.

¿Habría que admitir esas costumbres en nombre del respeto a la estructura interna de la comunidad? Un liberal no puede admitir que se viole la libertad del individuo en aras de salvaguardar la identidad del grupo, ni puede admitir que el individuo no tenga derecho a revisar las autoridades y prácticas tradicionales.

El término derechos colectivos resulta confuso porque hay diversas formas de ciudadanía diferenciada. «Los derechos diferenciados en función del grupo pueden ser otorgados a los miembros individuales de un grupo o al grupo como un todo, o a un Estado/provincia federal dentro del cual el grupo en cuestión constituye la mayoría» (p. 72). Son derechos diferenciados en función del grupo porque todos ellos se otorgan en función de la pertenencia cultural. Ejemplos que corresponden a esas situaciones diversas son por ejemplo, del primer caso los derechos de los canadienses de habla francesa a procesos judiciales en francés en cualquier parte del país a expensas del gasto público, o el derecho de un grupo de padres francófonos a exigir una escuela francesa allá donde el número de niños lo justifique, o el de grupos como un todo serían los derechos especiales de caza y pesca de los pueblos indígenas en Canadá. «La justicia entre grupos exige que a los miembros de grupos diferentes se les conceda derechos diferentes»» (p. 76).

En resumen, dice Kymlicka, «una perspectiva liberal exige libertad dentro del grupo minoritario, e igualdad entre los grupos minoritarios y mayoritarios» (p. 212). 


\section{La tolerancia y sus límites}

Las democracias liberales pueden acomodar y adoptar muchas formas de diversidad cultural pero no todas. Y este punto supone el límite de lo que un liberal puede tolerar, como expone en uno de los más esclarecedores capítulos de este libro. Un liberal no puede aceptar las restricciones internas, la idea de que resulta moralmente legítimo para un grupo oprimir a sus miembros en nombre de la solidaridad grupal, la ortodoxia religiosa o la pureza cultural. Tal conducta conculca el compromiso liberal con la autonomía individual. Pero también pone límites a las protecciones externas, cuando esto supone que un grupo oprima o explote a otro, como en el apartheid (pp. 211-212).

¿Se podría acusar por ello al liberalismo de sectario? No, porque nadie está autorizado en nombre de la defensa de la identidad del grupo a no respetar los derechos civiles o las libertades políticas de sus miembros.

Este límite no es imponer un tipo de cultura sobre otra sino respetar los derechos civiles. En definitiva no es más que respetar los derechos humanos que figuran positivizados en la mayoría de las constituciones del mundo. Los grupos deben respetar los derechos de sus miembros a la disidencia y también la capacidad crítica de replantearse sus propios valores, así como la libertad de conciencia de cada individuo, que no puede ser usurpada por el grupo. Cada persona racional y autónoma es libre de replantearse cuando quiera sus propias metas en la vida.

Por tanto, Kymlicka rechaza enfáticamente algunas de las propuestas de comunitaristas como Sandel en este punto. Según este último el individuo pertenece de un modo fijo a una comunidad cultural más allá de cualquier cuestionamiento racional.

\section{Convencer con el diálogo y la razón, pero no imponer por la fuerza}

Pero ¿qué hacer cuando una minoría nacional autogobernada adopta prácticas iliberales respecto a sus propios miembros? ¿Los Estados liberales deberían imponer el liberalismo a estas minorías ¡liberales? (pp. 22).

Sin embargo el hecho de que un liberal esté autorizado a juzgar como injustas determinadas situaciones no le autoriza a imponer de un modo coactivo sus principios ni siquiera a los que no respetan los derechos de los demás. «Tanto los Estados extranjeros como las minorías nacionales constituyen comunidades políticas distintas, con sus propios derechos al autogobierno. En ambos casos, los intentos de imponer los principios liberales por la fuerza se perciben como una forma de agresión o de colonialismo paternalista. Y, a consecuencia de ello, estos intentos acaban en un profundo fracaso» (pp. 230-23 1). Además la historia demuestra, como se ha visto en la descolonización en África, que esto es ineficaz. 
Una excepción discutible sería la intervención justificada en casos de violación sistemática de los derechos humanos, como la esclavitud, el genocidio, la tortura, las expulsiones masivas, limpieza étnica; pero ésta podría ser hecha sólo por un organismo internacional aceptado como la ONU, no por individuos o grupos de países (p. 229 y 233).

Esto tampoco justifica el conformismo. En una sociedad liberal se puede exigir a quienes se integran desde fuera a esa sociedad que asuman la obligación de cumplir con los derechos civiles. Pero en el caso de las minorías con autonomía que no hacen gala de liberalidad no se debe interferir coactivamente, pero se puede tratar de dialogar y utilizar vías racionales. «Esto no significa que los liberales deban quedarse al margen y no hacer nada. Una minoría nacional que gobierna de manera iliberal actúa injustamente, y los liberales tienen el derecho, y la responsabilidad, de manifestar su disconformidad ante esta injusticia. Por tanto, los reformistas liberales de estas culturas deberían intentar promover sus valores liberales, mediante las razones o el ejemplo, y los liberales ajenos a ellas deberían prestar su apoyo a todas las iniciativas del grupo encaminadas a liberalizar su cultura» (p. 231-232). En el caso de que sean otros países sólo será posible influir a través de mecanismos internacionales, pero esto nunca justifica la intervención.

\section{Inmigración y diversidad cultural}

Kymlicka analiza muy bien la situación americana, sobre todo de los países receptores de inmigración. Es un buen conocedor de la situación de Canadá, con sus minorías indias y el hecho diferencial de Quebec; de la inmigración en EE. UU. y del autogobierno de los portorriqueños, chicanos, indios americanos, de la problemática de los afroamericanos que merecen un tratamiento aparte; de los indígenas en Latinoamérica. Hay que distinguir los procesos de modernización en cualquier grupo cultural, del hecho de que las culturas indígenas tengan que desaparecer o del hecho de que tengan que ser obligadas a homogeneizarse adoptando la cultura mayoritaria.

$\mathrm{Su}$ actitud es muy respetuosa con la voluntad de los integrantes de los grupos culturales. En este punto hace gala de un liberalismo también coherente.

Aporta así un marco conceptual interesante desde el que se podrían examinar dos problemas típicamente europeos y que no se han dado en América. Uno es el de la confrontación del islamismo con la cultura liberal europea, fenómeno realmente preocupante en el Viejo Continente, y que puede desestabilizar la sociedad europea. El otro son los nacionalismos que pueden dar lugar a secesiones, no siempre pacíficas.

Kymlicka prudentemente afirma que cada caso merece un tratamiento diferente. Lo que es aplicable a una situación no lo es en otra. Así insiste en la diferente actitud de los grupos nacionales, que suelen reclamar su autogobierno, 
y la de los inmigrantes, que persiguen la integración dentro de las instituciones y del marco legal del país de acogida, lo que no es obstáculo para que quieran además mantener sus tradiciones y su identidad cultural.

La actitud de Kymlicka ante la inmigración y la diversidad cultural que ésta genera es altamente positiva. Ante el miedo que ha surgido en EE. UU. ante las reclamaciones de los derechos étnicos afirma Kymlicka que «El inevitable, y en el fondo deseable, hecho de los intercambios culturales no debilita la afirmación de que existen distintas culturas societales» (p. 105). Al contrario de lo que están haciendo muchos liberales en Estados Unidos, no propugna un sincretismo. Deja muy claro que la cultura americana, mal llamada melting pot, es en realidad una cultura anglosajona, no una síntesis de varias. Los inmigrantes europeos que fueron llegando se integraron en esta cultura. La vida política y las instituciones públicas y la enseñanza se hacían en inglés, existía una religión dominante (el cristianismo), obedecían a una cultura concreta, no eran neutrales, y hoy sigue siendo así.

Indudablemente se observa una evolución en la actitud y en la procedencia de los inmigrantes de EE. UU., a lo largo de este siglo. Los de la primera oleada fueron europeos y se fundieron en la cultura de los primitivos colonos de origen anglosajón. La segunda oleada está formada por orientales e hispanos, con un mayor afán de conservar sus raíces.

Otro problema diferente es el de los exiliados y refugiados que no es un fenómeno voluntario. En general estas personas ven su situación como provisional, aunque algunos acaban transformándose en inmigrantes.

En definitiva, un libro que invita a la reflexión serena, pionero y que introduce claridad en un debate muchas veces confuso. 
DOXA 20 (1997) 